\title{
Manejo da Adubação Nitrogenada de Tensões Hídricas sobre a Produção de Matéria Seca e Índice de Área Foliar de Tifton 85 Cultivado no Cerrado
}

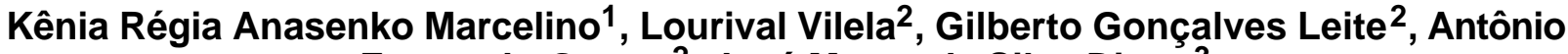 \\ Fernando Guerra ${ }^{2}$, José Mauro da Silva Diogo ${ }^{3}$
}

RESUMO - Este trabalho foi conduzido na Embrapa Cerrados, Planaltina (DF), objetivando avaliar a influência de tensões hídricas $(35,60,100$ e $500 \mathrm{kPa})$ e doses de nitrogênio (0 - Testemunha, 45, 90, 180, $360 \mathrm{~kg} / \mathrm{ha}$ ) sobre a produção de matéria seca (MS), a taxa de produção de matéria seca (TPMS) e o índice de área foliar (IAF) do capim Cynodon spp. cv. Tifton 85. Empregou-se delineamento em blocos ao acaso, com parcelas subdivididas em três repetições; nas parcelas foram distribuídas as tensões e nas subparcelas, as doses de nitrogênio $(\mathrm{N})$. As doses de nitrogênio influenciaram a produção de matéria seca em todas as tensões, onde a maior produtividade (41,8 t/ha) foi obtida na tensão de $35 \mathrm{kPa}$ e na dose de $360 \mathrm{~kg} / \mathrm{ha}$ de $\mathrm{N}$ e menor produtividade (17,3 t/ha), na tensão de $100 \mathrm{kPa}$ sem adição de N. Nas tensões de 60, 100 e $500 \mathrm{kPa}$, a eficiência na utilização do N foi maior nas doses mais elevadas. As maiores TPMS foram observadas no período com temperaturas elevadas, enquanto as menores foram no período com temperaturas reduzidas. Durante o período de 16/03 a 09/08, não se observou efeito das doses de N. Nos demais períodos, a TPMS aumentou com o incremento das doses de N. Não foram observados efeitos das tensões hídricas sobre a TPMS. O IAF elevou-se com o aumento nas doses de N, principalmente no período de 21/09 a 10/01. Entretanto nos períodos de baixa temperatura não houve respostas do IAF às doses crescentes de N. As tensões hídricas não afetaram o IAF em nenhum dos períodos avaliados.

Palavras-chave: gramínea, irrigação, nitrogênio, produtividade, taxa de produção de matéria seca

\section{Management of Nitrogen Fertilization and of Water Tension on the Production of Dry Matter and Leaf Area Index of Tifton 85 Cultivated in the Cerrado Region}

\begin{abstract}
This work was carried out to evaluate the influence of water tensions $(35,60,100$ and $500 \mathrm{kPa})$ and nitrogen levels (0 - Control, 45, 90, 180, $360 \mathrm{~kg} / \mathrm{ha}$ ) on the dry matter (DM) yield, the dry matter yield rate (DMYR) and the leaf area index (LAI) of Cynodon spp. cv. Tifton 85 bermudagrass. A randomized blocks design in a split splot scheme with three replications was used, split plots were represented by the water tensions were and the plots, by the nitrogen $(\mathrm{N})$ levels. The dry matter yield was influenced by the nitrogen levels for all tensions, where the highest productivity $(41.8 \mathrm{t} / \mathrm{ha})$ was obtained at $35 \mathrm{kPa}$ the tension and the maximum $\mathrm{N}$ level $(360 \mathrm{~kg} / \mathrm{ha}$ of $\mathrm{N})$, while the smallest productivity $(17.3 \mathrm{t} / \mathrm{ha})$, at $100 \mathrm{kPa}$ the tension without $\mathrm{N}$ addition. At the tensions of 60 , 100 and $500 \mathrm{kPa}$ there was limitation in the use of $\mathrm{N}$ at the highest doses, due to the deficit water. Higher DMAR were observed from $09 / 21$ to $11 / 29$, and the averages ranged from 73.4 to $194.6 \mathrm{~kg} / \mathrm{ha}$ /day of DM, while from 05/26 to 08/09 the smallest averages were observed, that ranged from 44.9 to $55.2 \mathrm{~kg} / \mathrm{ha} /$ day of DM. No effect of N levels was observed from 03/16 to 08/09. In the other periods, DMYR increased as N levels increased. There was no effect of the different water tensions on DMYR. LAI increased as N levels increased, from 09/21 to 01/10, with averages from 3.07 to 9.64. In the periods with low temperatures, N levels and did not affect LAI. Water tension did not affect LAI in all evaluated periods.
\end{abstract}

Key Words: dry matter yield rate, forage accumulation rate, grass, irrigation, nitrogen, productivity

\section{Introdução}

As pastagens naturais na região do Cerrado são caracterizadas por gramíneas de baixo valor nutritivo e baixa produtividade, onde predominam solos ácidos e com baixa fertilidade. Considerando que a base de sustentação da alimentação bovina da região consiste na utilização de pastagens, existe a necessidade de elevar o potencial de produção forrageira. Para atender este objetivo, foram introduzidas gramíneas tropicais melhoradas e adaptadas, que apresentam maior produtividade do que as gramíneas nativas, especialmente, mediante a adoção de práticas de manejo associadas a intensificação no uso de insumos.

Entre as gramíneas cultivadas na região do Cerrado, está o capim Tifton 85 (Cynodon spp.), que

\footnotetext{
${ }^{1}$ Estudante de doutorado, Departamento de Zootecnia, UFV, Viçosa, MG. E.mail: kramarcelino@yahoo.com.br

2 Pesquisadores de EMBRAPA Cerrados, Brasília, DF. E.mail: sac@cpac.embrapa.br

3 Professor Adjunto da Faculdade de Agronomia e Medicina Veterinária da UnB, Brasília, DF. E.mail: diogojm@unb.br
} 
teve sua origem na Coastal Plain Experiment Station (USDA-University of Geórgia), no sul do Estado da Geórgia (Burton et al., 1993). Este cultivar, conhecido como grama bermuda foi selecionado por sua elevada produção de matéria seca e boa digestibilidade em comparação a maioria das gramas bermudas híbridas (Pedreira, 1996).

A utilização de adubação em pastagens, particularmente a nitrogenada, é prática fundamental quando se pretende aumentar a produção de matéria seca, pois o nitrogênio $(\mathrm{N})$ presente no solo, proveniente da mineralização da matéria orgânica derivada do complexo solo-planta-animal, não é suficiente para as gramíneas de alta produção expressarem o seu potencial (Guilherme et al., 1995). Vários trabalhos reportam aumento da produtividade com a utilização de adubação nitrogenada (Corsi, 1994; Nabinger, 1997). Fernandez et al. (1989), trabalhando com Cynodon spp. (Coastcross), obtiveram produções de 12,0 t/ha no tratamento sem nitrogênio, enquanto no tratamento com $600 \mathrm{~kg} / \mathrm{ha}$ de $\mathrm{N}$ a produção foi de $35,4 \mathrm{t} / \mathrm{ha}$ de MS, apresentando eficiência de $39 \mathrm{~kg}$ $\mathrm{MS} / \mathrm{kg}$ de $\mathrm{N}$ aplicado. Respostas lineares em produção em resposta à adubação nitrogenada têm sido observadas até doses de $600 \mathrm{~kg} / \mathrm{ha}$ de $\mathrm{N}$, todavia, sua eficiência na utilização pela planta nos níveis mais elevados é dependente da umidade, proveniente da irrigação ou das chuvas (Vilela \& Alvim, 1998).

Plantas sob déficit hídrico sofrem mudanças em sua anatomia, fisiologia e bioquímica, com intensidade que depende do tipo de planta e do grau de duração do déficit hídrico (Kramer, 1983). A primeira estratégia da planta para se adaptar às condições de estresse hídrico é a redução da parte aérea em favor das raízes, limitando sua capacidade de competir por luz, pela diminuição da área foliar, com conseqüente redução na produtividade (Nabinger, 1997). Fernandez et al. (1986) obtiveram respostas em capim-coastcross irrigado até a dose de $675 \mathrm{~kg} / \mathrm{ha}$ de $\mathrm{N}$ na época chuvosa e na seca, até a dose de $225 \mathrm{~kg} / \mathrm{ha}$ de N. Observaram ainda que, mesmo com irrigação no período seco, o potencial de produção da forrageira é limitado, provavelmente devido a fatores climáticos, como luminosidade e temperatura incidentes neste período. Pedreira et al. (1998) reportam que, em regiões com latitudes superiores a $25^{\circ} \mathrm{S}$, pode ocorrer redução do período de crescimento, quando comparado com localidades mais próximas ao equador, onde, durante o inverno, altas temperaturas podem justificar economicamente a utilização da irrigação para a produção de Cynodon.
O índice de área foliar (IAF), que foi descrito por Watson como a relação entre a área foliar e a área de solo que essas folhas ocupam, possibilita melhor entendimento das relações entre a interceptação de luz e o acúmulo de forragem. Maior IAF proporciona aumento da luz interceptada, no entanto, o aumento demasiado provocará o auto-sombreamento, acarretando diminuição na taxa fotossintética média por unidade de área foliar, em razão da maior resistência imposta pelos estômatos e mesófilo foliar à absorção do $\mathrm{CO}_{2}$ (Gomide, 1994).

$\mathrm{Na}$ estação de crescimento, a produtividade, eficiência fotossintética e IAF das plantas aumentam, podendo alcançar o valor crítico em que $95 \%$ da luz incidente é interceptada e a máxima taxa de crescimento da cultura é obtida. Oliveira et al. (2000) observaram que o IAF crítico para o capim-Tifton 85 está em torno de 4,9 aos 45 dias de idade. Entretanto, a relação entre fixação de carbono e produção de matéria seca pode sofrer a ação de outros fatores de produção, tais como atividade dos drenos metabólicos e/ou equilíbrio entre produção e perdas, como senescência, respiração e pastejo (Gomide, 1996).

A prática de irrigação em pastagens na região do Cerrado vem sendo utilizada, sem embasamento científico de forma empírica, sendo necessário definir estratégias de manejo que otimizem a produção das espécies tropicais submetidas à adubação nitrogenada e irrigação. O objetivo deste trabalho foi verificar a influência de diferentes doses de nitrogênio e de tensões hídricas no solo sobre a produção de matéria seca e o índice de área foliar do capim Cynodonspp. cv. Tifton 85 .

\section{Material e Métodos}

O trabalho foi conduzido no período de março de 2000 a janeiro de 2001 em uma área de Latossolo Vermelho Escuro, textura argilosa, na Embrapa Cerrados, em Planaltina/DF, localizado a $1007 \mathrm{~m}$ de altitude, a $15^{\circ} 35^{\prime} 30^{\prime \prime}$ de latitude Sul e 47\%2'30" de longitude Oeste.

A precipitação pluviométrica total no período experimental foi de $799,5 \mathrm{~mm}$ e as temperaturas médias máxima e mínima, de 28 e $16^{\circ} \mathrm{C}$, respectivamente (Figura 1).

A análise do solo, na camada de 0 a $20 \mathrm{~cm}$, apresentou as seguintes características químicas: $\mathrm{pH}$ $\left(\mathrm{H}_{2} \mathrm{O}, 1: 2,5\right)=5,40 ; \mathrm{Al}=0,03 \mathrm{cmolc} / \mathrm{dm}^{3} ; \mathrm{Ca}+\mathrm{Mg}=$ $4,58 \mathrm{cmolc} / \mathrm{dm}^{3} ; \mathrm{K}=373,0 \mathrm{mg} / \mathrm{dm}^{3} ; \mathrm{P}=59,9 \mathrm{mg} / \mathrm{dm}^{3}$; 


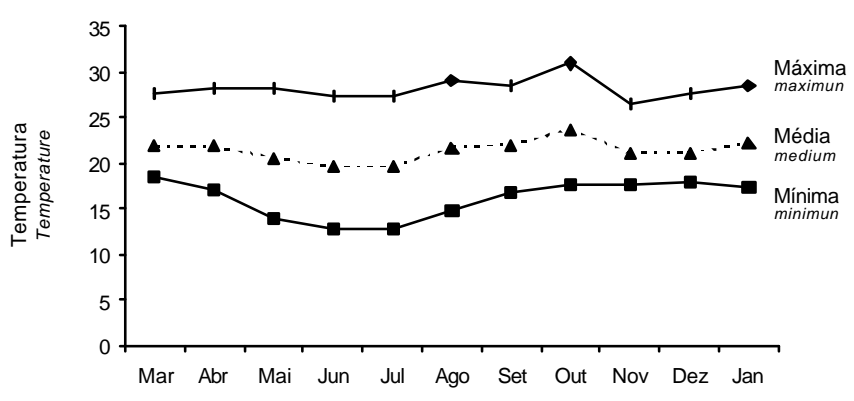

Figura 1 - Temperaturas máximas, médias e mínimas (média mensal) durante o período experimental (março/2000 a janeiro/2001).

Figure 1 - Maximum, medium and minimum temperatures (monthly average) during the experimental period (from March/2000 to January 2001).

$\mathrm{H}+\mathrm{Al}=4,94 \mathrm{cmolc} / \mathrm{dm}^{3}$, matéria orgânica $=24,6 \mathrm{~g} / \mathrm{dm}^{3}$ e saturação por bases $=53 \%$.

Os tratamentos consistiram de quatro tensões hídricas $(35,60,100$ e $500 \mathrm{kPa})$ e cinco doses de nitrogênio (0-Testemunha, 45, 90, 180 e 360 kg/ha) em parcelas estabelecidas de capim Cynodon spp. cv. Tifton 85, que foram plantadas em novembro de 1999. A fonte de nitrogênio utilizada foi a uréia, parcelada em quatro aplicações, nos meses de março, maio, agosto e novembro (Tabela 1). A adubação de plantio constou de $40 \mathrm{~kg} / \mathrm{ha}$ de $\mathrm{P}_{2} \mathrm{O}_{5}$ e a de manutenção, de $400 \mathrm{~kg} / \mathrm{ha}$ de 00-25-25 $\left(\mathrm{N}-\mathrm{P}_{2} \mathrm{O}_{5^{-}}\right.$ $\mathrm{K}_{2} \mathrm{O}$ ), aplicado após seis meses.

Utilizou-se delineamento experimental de blocos ao acaso com parcelas subdivididas e três repetições. Cada bloco foi composto de quatro parcelas, nas quais foram testadas as tensões hídricas e nas subparcelas as doses de nitrogênio.

A irrigação foi realizada por microaspersão com vazão de 0,28 L/s. Após a emergência das plântulas, foram instalados tensiômetros nas profundidades de $15,30,45,60$ e $75 \mathrm{~cm}$ e blocos de gesso a 15 e $30 \mathrm{~cm}$. As leituras nos tensiômetros e blocos de gesso foram feitas diariamente pela manhã. $\mathrm{O}$ momento de irrigação foi determinado quando a tensão de água no solo, na profundidade de $15 \mathrm{~cm}$ atingiu os valores correspondentes a cada tratamento, estabelecendo-se as
Tabela 1 - Datas e doses de nitrogênio (N-uréia) aplicadas Table 1 - Dates and N levels (N-urea)

\begin{tabular}{llcccc}
\hline \multirow{2}{*}{$\begin{array}{l}\text { Data } \\
\text { Date }\end{array}$} & \multicolumn{5}{c}{$\begin{array}{l}\text { Dose de N (kg/ha) } \\
\text { N level (kg/ha) }\end{array}$} \\
\cline { 2 - 6 } & 0 & 45 & 90 & 180 & 360 \\
\hline $29 / 03 / 00$ & 0 & 7,5 & 15 & 30 & 60 \\
$31 / 05 / 00$ & 0 & 7,5 & 15 & 30 & 60 \\
$16 / 08 / 00$ & 0 & 15 & 30 & 60 & 120 \\
$01 / 11 / 00$ & 0 & 15 & 30 & 60 & 120 \\
\hline
\end{tabular}

lâminas brutas de água para irrigação para a camada de 0 a $35 \mathrm{~cm}$.

Subseqüentemente ao corte de uniformização $(16 / 03 / 00)$, as parcelas $\left(4 \mathrm{~m} \times 5 \mathrm{~m}=20 \mathrm{~m}^{2}\right)$ foram submetidas a cinco cortes, para a avaliação da taxa de produção de matéria seca. As datas de corte, precipitação e água fornecida via irrigação, são apresentados na Tabela 2 .

As variáveis avaliadas foram produção de matéria seca (MS), taxa de produção de matéria seca (TPMS) e índice de área foliar (IAF).

A matéria verde foi amostrada a $10 \mathrm{~cm}$ do solo, compreendida em dois quadrados de $50 \mathrm{~cm}$ de lado, dispostos aleatoriamente em cada unidade experimental e posteriormente seca em estufa com circulação de ar forçada por 72 horas a $60^{\circ} \mathrm{C}$.

Mediante as produções de MS em cada período, foram calculadas as taxas de produção de matéria seca, utilizando-se a seguinte fórmula:

$$
\text { TPMS }=\left(\mathrm{MS}_{2}-\mathrm{MS}_{1}\right) /\left(\mathrm{T}_{2}-\mathrm{T}_{1}\right)
$$

em que $\mathrm{MS}_{1}$ e $\mathrm{MS}_{2}$ são os pesos da matéria seca amostrados em dois tempos sucessivos e $\mathrm{T}_{1}$ (tempo inicial) e $\mathrm{T}_{2}$ (tempo final).

Para determinação do IAF, foram tomadas leituras em todas as parcelas antes do corte, utilizando-se um analisador de dossel LAI - 2000 da LI-COR. Foi estabelecido um fator de correção $(4,26)$, que foi obtido por intermédio dos índices de um integrador de área foliar HAYASHI DENKOH (modelo AA - C400). No período de 16/03 a 24/05/00, não foram tomadas as medidas de IAF, devido a problemas no aparelho.

As análises estatísticas e o ajuste das equações de regressão foram feitos utilizando-se o aplicativo STATISTICAL ANALYSIS SYSTEM, sendo feitas comparações de médias pelo teste Tukey. 
Tabela 2 - Datas de corte, precipitação pluviométrica e quantidade de água fornecida via irrigação durante o período experimental

Table 2 - Cutting date, rainfall and water amount fed by irrigation during the experimental period

\begin{tabular}{|c|c|c|c|c|c|}
\hline \multirow[t]{2}{*}{$\begin{array}{l}\text { Data do corte } \\
\text { Cutting date }\end{array}$} & \multirow[t]{2}{*}{$\begin{array}{c}\text { Precipitação }^{1}(\mathrm{~mm}) \\
\text { Rainfall }^{1}\end{array}$} & \multicolumn{4}{|c|}{$\begin{array}{l}\text { Àgua fornecida }^{1}(\mathrm{~mm}) \\
\text { Water supplied }(\mathrm{mm})\end{array}$} \\
\hline & & $35 \mathrm{kPa}$ & $60 \mathrm{kPa}$ & $100 \mathrm{kPa}$ & $500 \mathrm{kPa}$ \\
\hline \multicolumn{6}{|l|}{$16 / 03 / 00$} \\
\hline $25 / 05 / 00$ & 95,8 & $141,5(9)^{2}$ & $124,8(13)$ & $104,4(21)$ & $41,1(50)$ \\
\hline 09/08/00 & 1,7 & $206,9(7)$ & $184,0(9)$ & 169,5 (14) & $123,0(21)$ \\
\hline $20 / 09 / 00$ & 94,0 & 62,2 (12) & 94,0 (14) & $63,1(15)$ & 80,2 (16) \\
\hline $29 / 11 / 00$ & 348,1 & $129,5(11)$ & $129,0(13)$ & $132,1(16)$ & 80,7 (30) \\
\hline $10 / 01 / 01$ & 259,9 & $38,0(21)$ & 0 & 0 & 0 \\
\hline Total & 799,5 & 578,1 & 531,8 & 469,5 & 325,0 \\
\hline
\end{tabular}

1 Total do período.

2 Valores entre parênteses referem-se ao intervalo médio entre irrigação em dias.

1 Total of period.

2 Values within brackets refer to the average irrigation frequency in days.

\section{Resultados e Discussão}

O acúmulo total de matéria seca (MS) em relação às doses de nitrogênio $(\mathrm{N})$ foi avaliado para cada uma das tensões hídricas (Figura 2). A maior produção obtida foi na menor tensão hídrica $(35 \mathrm{kPa})$ e na dose máxima de $\mathrm{N}$, enquanto a menor foi encontrada na tensão de $100 \mathrm{kPa}$ e sem adubação nitrogenada.

$\mathrm{Na}$ tensão hídrica de $35 \mathrm{kPa}$, a resposta de produção foi linear em relação a doses de $\mathrm{N}$, o que se atribui à eficiência no aproveitamento do $\mathrm{N}$ em condições de maior umidade no solo. A eficiência no aproveitamento de $\mathrm{N}$ foi de $36 \mathrm{~kg} \mathrm{MS} / \mathrm{kg}$ de $\mathrm{N}$ aplicado, bem próximo ao obtido por Ribeiro (2000) 36,8 kg MS/kg de N, enquanto que Fernandez et al. (1989) trabalhando com capim-coastcross obtiveram aumentos variando de 39,25 a 51,35 kg MS/kg N aplicado.

$\mathrm{Na}$ tensão de $60 \mathrm{kPa}$, a resposta foi assintótica, com incrementos na produção até a dose de $90 \mathrm{~kg} / \mathrm{ha}$ de N. A produção mais elevada nesta tensão também foi obtida na maior dose de $\mathrm{N}$ (360 kg/ha), todavia, os incrementos de MS a partir de $90 \mathrm{~kg} / \mathrm{ha}$ de $\mathrm{N}$ foram bastante reduzidos. Com a aplicação de 45, 90, 180 e $360 \mathrm{~kg} /$ ha de $\mathrm{N}$, houve aumento na produção de 23,5 ; 26,$9 ; 28,7$ e $32,7 \%$ em relação à dose zero, respectivamente, evidenciando redução no acréscimo de MS produzida, provavelmente imposta pelo déficit hídrico no aproveitamento do $\mathrm{N}$.

Respostas inferiores às observadas nas tensões de 35 e $60 \mathrm{kPa}$ ocorreram nas de $100 \mathrm{kPa}$ e $500 \mathrm{kPa}$, obtendo-se com $500 \mathrm{kPa}$ maiores produções que na

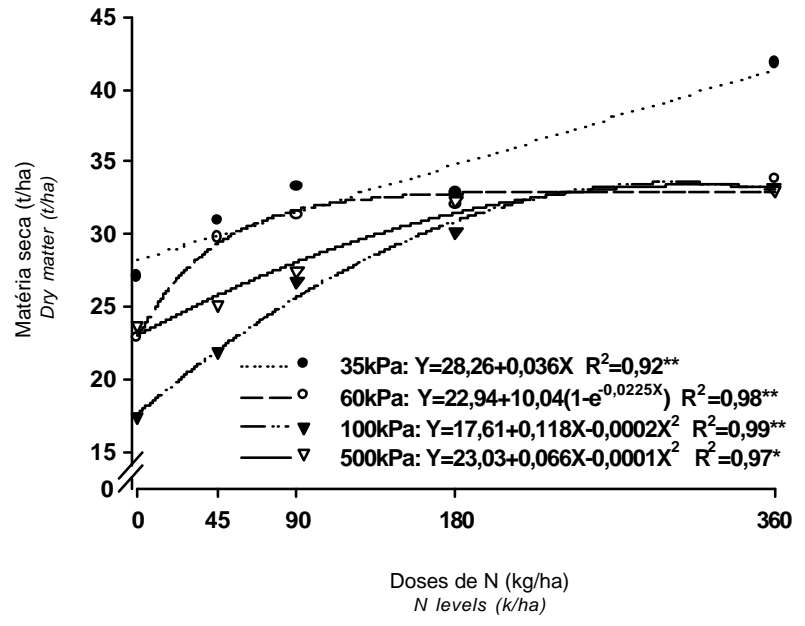

Figura 2 - Produção de matéria seca (t/ha) por tensões hídricas em Cynodon spp. cv. Tifton 85, nas diferentes doses de nitrogênio.

Figure 2 - Dry matter yield (t/ha) by water tension in Cynodon spp. cv. Tifton 85, under different nitrogen levels.

de $100 \mathrm{kPa}$, mesmo para níveis mais baixos de N. Já o acúmulo de matéria seca natensão de $500 \mathrm{kPa}$ foi de 23,$3 ; 25,8 ; 28,2 ; 31,7$ e 33,8 tha, na de $100 \mathrm{kPa}$ foi observada produção de 17,6; 22,5; 26,6; 32,4 e 34,1 t/ha de MS nas doses de 0, 45, 90, 180 e $360 \mathrm{~kg} / \mathrm{ha}$ de $\mathrm{N}$, respectivamente.

Nas tensões de 100 e $500 \mathrm{kPa}$, as respostas foram quadráticas, com incrementos na produção de MS 
respectivamente até a dose de 295 e $330 \mathrm{~kg} / \mathrm{ha}$ de $\mathrm{N}$. A partir destas doses, houve redução na produção, evidenciando a influência de outros fatores, provavelmente a falta de água para o aproveitamento do nitrogênio fornecido. Além da alteração nos processos morfológicos e fisiológicos na planta sob estresse hídrico, há redução na absorção de nutrientes como o nitrogênio (Norton, 1982). A quantidade total de água fornecida via irrigação na tensão de $100 \mathrm{kPa}$ foi de $469,5 \mathrm{~mm}$, enquanto na tensão de $500 \mathrm{kPa}$ foi de $325,0 \mathrm{~mm}$, ou seja, redução de 19 e $44 \%$, quando comparada com a tensão de $35 \mathrm{kPa}$, que foi de $578,1 \mathrm{~mm}$ (Tabela 2). Trabalhos com capimcoastcross reportam aumentos progressivos na produção de forragem até doses de 400 - $600 \mathrm{~kg} / \mathrm{ha} \mathrm{de} \mathrm{N}$ (Fernandez et al., 1989; Alvim et al., 1998). Sem a irrigação, no entanto, a resposta aconteceu somente no período chuvoso, caracterizando dependência de água para aumento na eficiência de uso do N. Trabalhos realizados com milho mostraram maiores produções com a elevação nas doses de $\mathrm{N}$ e com tensões variando entre 33 e $100 \mathrm{kPa}$. Entretanto, doses acima de $180 \mathrm{~kg} / \mathrm{ha}$ de $\mathrm{N}$ só aumentaram a produtividade sob menores tensões hídricas, sendo que tensões elevadas ocasionaram redução na produtividade (Guerra et al., 1997).

De forma geral, com a tensão de água no solo em $35 \mathrm{kPa}$, ocorreram incrementos satisfatórios na produção de matéria seca até a dose de $360 \mathrm{~kg} / \mathrm{ha}$ de N. Nas tensões de 60, 100 e 500 kPa houve incrementos até a dose de $360 \mathrm{~kg} / \mathrm{ha}$ de $\mathrm{N}$, entretanto maiores aumentos ocorreram até as doses de 90,180 e $180 \mathrm{~kg} / \mathrm{h}$ a de $\mathrm{N}$, nas tensões de 60,100 e $500 \mathrm{kPa}$, respectivamente.

A taxa de produção de matéria seca (TPMS), foi diferente $(\mathrm{P}<0,01)$ entre os períodos de corte, apresentando menores valores no período de 26/05 a 09/ 08, variando de 44,90 a 57,29 kg/ha/dia de MS e maiores no período de 21/09 a 29/11, variando de 73,41 a 194,65 kg/ha/dia de MS (Tabela 3).

Observou-se interação entre período de corte e tensões hídricas $(\mathrm{P}<0,01)$, porém não houve efeito de tensão dentro de cada período $(\mathrm{P}>0,05)$ para TPMS. Houve diferença na TPMS em relação às doses de $\mathrm{N}$

Tabela 3 - Taxa de produção de matéria seca (kg/ha/dia) de Cynodon spp. cv. Tifton 85 nos cinco períodos avaliados, nas doses de nitrogênio e tensões hídricas testadas ${ }^{1}$

Table 3 - Dry matter yield rate ( $\mathrm{kg} / \mathrm{ha} /$ day DM) of Cynodon spp. cv. Tifton 85, in the five evaluated periods, under different nitrogen levels and water tensions

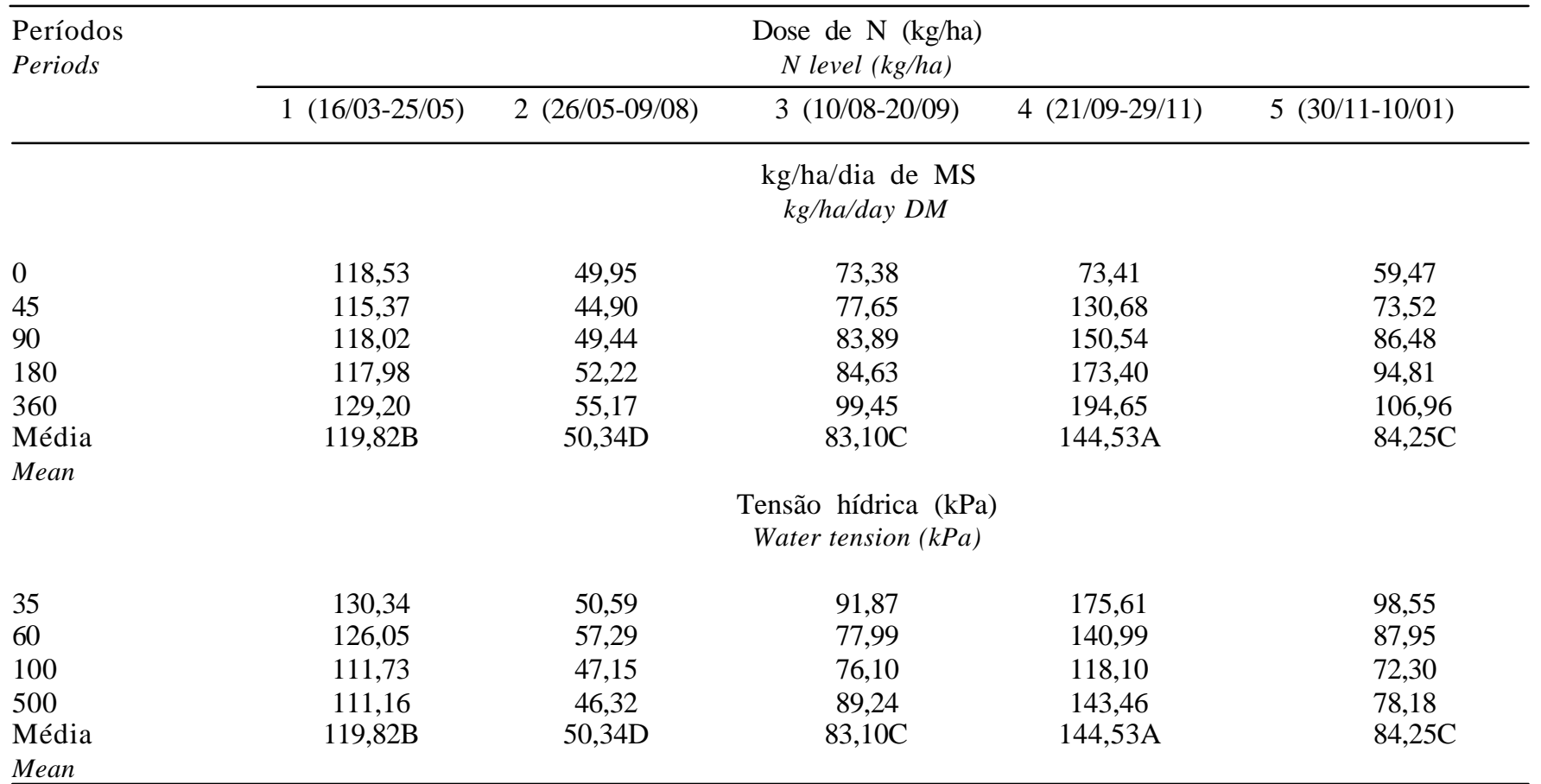

${ }^{1}$ Médias seguidas de letras iguais na linha não diferem pelo teste de Tukey a $5 \%$ de probabilidade.

${ }^{1}$ Means, within a row, followed by the same letter differ by Tukey test at $5 \%$ of probability. 
$(\mathrm{P}<0,01)$ e interação entre período de corte e doses de $\mathrm{N}(\mathrm{P}<0,05)$. Isto provavelmente pode ser atribuído à influência das condições climáticas, principalmente temperatura e insolação, na eficiência de utilização de $\mathrm{N}$ e de água, sendo que a ocorrência de déficit hídrico durante o crescimento das plantas, pode ter promovido menor eficiência em aproveitamento, tanto da energia solar quanto da adubação nitrogenada, conseqüentemente menor TPMS. Foram ajustadas equações de regressão para as TPMS nas diferentes doses de N, nos cinco períodos de avaliação (Figura3).

Nos períodos de 16/03 a 25/05 e 26/05 a 09/08, não foram observados efeitos das doses de $\mathrm{N}(\mathrm{P}>0,05)$. Neste último (26/05 a 09/08) foram observadas as menores temperaturas do período experimental, que podem ter ocasionado menores TPMS. Neste período, as condições climáticas foram limitantes ao cresci-

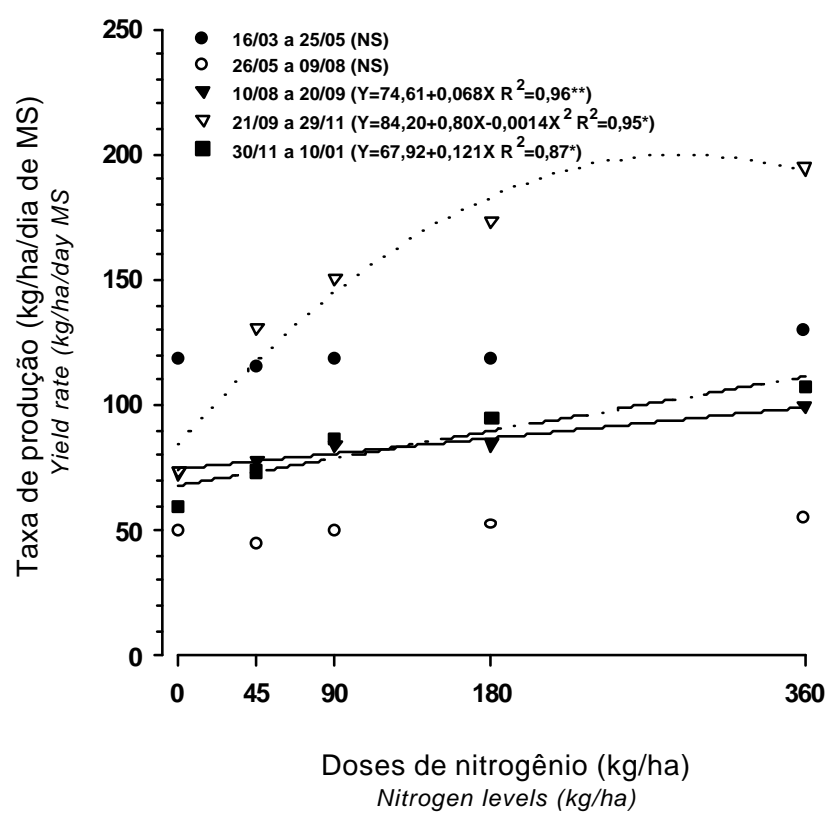

Figura 3 - Taxa de produção de matéria seca $(\mathrm{kg} / \mathrm{ha} / \mathrm{dia}$ de MS) do Cynodon spp. cv. Tifton 85 nas diferentes doses de nitrogênio, nos cinco períodos de avaliação.

Figure 3 - Dry matter yield rate $(\mathrm{kg} / \mathrm{ha} /$ day DM) of Cynodon spp. cv. Tifton 85, under different nitrogen levels, in the five evaluated periods. mento, onde a utilização de adubação nitrogenada e de irrigação não exerceram influência na TPMS. Langer (1963) reporta que os principais fatores ambientais que afetam o perfilhamento são a temperatura, a luz, a nutrição mineral e o suprimento de água.

Aumentos lineares na TPMS com o incremento nas doses de $\mathrm{N}$ foram observados no período de 10/08/00 a 20/09 e de 30/11 a 10/01/01. No período de 21/09 a 29/11 ocorreram as maiores TPMS e taxa máxima estimada foi obtida com a aplicação de $286 \mathrm{~kg} / \mathrm{ha}$ de $\mathrm{N}$. Isso pode ser atribuído às condições climáticas satisfatórias do período. As temperaturas elevadas e a luminosidade do período, podem ter favorecido o aumento na produção, devido à maior eficiência na utilização de $\mathrm{N}$, principalmente nos tratamentos com maiores tensões.

Houve efeito $(\mathrm{P}<0,05)$ de doses de $\mathrm{N}$ sobre o IAF, somente nos períodos de 21/09 a 29/11 e 30/11 a 10/01 (Tabela 4). No período de 21/09 a 29/11 o IAF variou de 6,3 a 9,6 e de 3,1 a 5,8 no período de 30/11 a $10 / 01$, nos tratamentos sem nitrogênio e com $360 \mathrm{~kg} / \mathrm{ha}$ de $\mathrm{N}$, respectivamente.

Não houve $(\mathrm{P}>0,05)$ efeito de tensões hídricas sobre o IAF em nenhum dos períodos avaliados. Entretanto, Nabinger (1997) informou que plantas submetidas a déficit hídrico apresentam redução do IAF, devido à redução da fotossíntese e do alongamento de folhas. Em trabalhos realizados com milho, França et al. (1999) verificaram que a ocorrência de déficit hídrico durante o crescimento vegetativo ocasionou atraso nos estádios fisiológicos seguintes, afetando negativamente o IAF.

Houve diferença no IAF $(\mathrm{P}<0,05)$ com relação aos períodos de corte, o que pode ser atribuído aos diferentes intervalos de corte das plantas. No período de 21/09 a 29/11, foram observados maiores IAF. Isto pode ter ocorrido devido à aplicação de nitrogênio do período ter sido realizada sob condições de temperatura e luminosidade favoráveis, o que estimulou o desenvolvimento de área foliar. Trabalhando com Tifton 85 aos 14 e 49 dias de idade, respectivamente, Gomide (1996) encontrou valores de IAF variando de 1,68 a 5,85. Oliveira (2000) encontrou 4,9 como sendo o IAF crítico no Tifton 85, aproximadamente aos 45 dias de rebrota. 
Tabela 4 - Índice de área foliar do Cynodon spp. cv. Tifton 85 em quatro períodos avaliados, nas doses de nitrogênio e tensões hídricas testadas ${ }^{1}$

Table 4 - Leaf area index of Cynodon spp. cv. Tifton 85, in the four evaluated periods, under different nitrogen levels and water tensions

\begin{tabular}{|c|c|c|c|c|}
\hline & \multicolumn{4}{|c|}{$\begin{array}{c}\text { Dose de } \mathrm{N}(\mathrm{kg} / \mathrm{ha}) \\
N \text { level }\end{array}$} \\
\hline & Período 2 (26/05-09/08) & Período 3 (10/08-20/09) & Período 4 (21/09-29/11) & Período $5(30 / 11-10 / 01)$ \\
\hline & \multicolumn{4}{|c|}{ IAF } \\
\hline 0 & $5,54 \mathrm{a}$ & $4,28 \mathrm{a}$ & $6,30 \mathrm{~b}$ & $3,19 b$ \\
\hline 45 & $5,37 \mathrm{a}$ & $4,59 a$ & $8,26 \mathrm{ab}$ & $3,55 b$ \\
\hline 90 & $6,57 \mathrm{a}$ & $5,38 \mathrm{a}$ & $9,17 \mathrm{ab}$ & $4,14 a b$ \\
\hline 180 & $5,98 \mathrm{a}$ & $5,35 \mathrm{a}$ & $9,03 \mathrm{ab}$ & $4,69 \mathrm{ab}$ \\
\hline \multirow[t]{2}{*}{360} & $6,24 \mathrm{a}$ & $5,49 \mathrm{a}$ & $9,64 \mathrm{a}$ & $5,77 \mathrm{a}$ \\
\hline & \multicolumn{4}{|c|}{$\begin{array}{l}\text { Tensão hídrica (kPa) } \\
\text { Water tension }\end{array}$} \\
\hline 35 & $5,77 \mathrm{a}$ & $5,44 \mathrm{a}$ & $9,13 \mathrm{a}$ & $4,75 \mathrm{a}$ \\
\hline 60 & $6,64 a$ & $4,90 \mathrm{a}$ & $8,98 \mathrm{a}$ & $4,23 a$ \\
\hline 100 & $5,38 \mathrm{a}$ & $4,45 \mathrm{a}$ & $7,03 a$ & $4,07 \mathrm{a}$ \\
\hline 500 & $5,98 \mathrm{a}$ & $5,29 \mathrm{a}$ & $8,72 \mathrm{a}$ & $3,94 a$ \\
\hline
\end{tabular}

${ }^{1}$ Médias seguidas de letras iguais na coluna não diferem pelo teste de Tukey, a $5 \%$ de probabilidade.

${ }^{1}$ Means, within a column followed by the same letter do not differ by Tukey test at $5 \%$ of probability.

\section{Conclusões}

Nas condições em que o trabalho foi desenvolvido, a dose de $360 \mathrm{~kg} / \mathrm{ha}$ de $\mathrm{N}$ na tensão hídrica no solo de $35 \mathrm{kPa}$ proporcionou maior produção de matéria seca nos períodos com condições climáticas não limitantes.

Nos períodos com condições climáticas favoráveis, o aumento nas doses de $\mathrm{N}$ aplicadas proporcionou incrementos no IAF.

\section{Literatura Citada}

ALVIM, M.J.; XAVIER, D.F.; BOTREL, M.A. et al. Resposta do coast-cross (Cynodon dactylon (L) Pers.) a diferentes doses de nitrogênio e intervalos de cortes. Revista Brasileira de Zootecnia, v.27, n.5, p.833-840, 1998.

BURTON, G.W.; GATES, R.N.; HILL, G.M. Registration of "Tifton 85" bermudagrass. Crop Science, v.33, p.644-645, 1993.

CORSI, M. Adubação nitrogenada das pastagens. In: PEIXOTO, A.M.; MOURA, J.C.; FARIA, V.P. (Eds.)Pastagens: Fundamentos da exploração racional. Piracicaba: Fundação de Estudos Agrários Luiz de Queiroz, 2.ed. 1994. p.121-153.

FERNANDEZ, D.; GOMEZ, I.; PARETAS, J.J. Fertilización nitrogenada en bermuda cruzada n. 1 (Cynodon dactylon) sobre suelo pardo tropical. Pastos y Forrajes, v.6, n.1, p.27-49, 1986.

FERNANDEZ, D.; PARETAS, J.J.; FONSECA, E. Influencia de la fertilización com nitrogeno y la frecuencia de corte en bermuda cruzada 1 (coast-cross 1) com riego e sin el. 1. Rendimento e economia. Pastos y Forrajes, v.12, n.1, p.41-55, 1989.
FRANÇA, S. BERGAMASCHI, H e ROSA, L.M.G. Modelagem do crescimento em função da radiação fotossinteticamente ativa e do acúmulo de graus-dia, com e sem irrigação.Revista Brasileira de Agrometeorologia, v.7, n.1, p.59-66, 1999. GOMIDE, C.C.C. Algumas características fisiológicas e químicas de cinco cultivares de Cynodon. Jaboticabal: Universidade Estadual Paulista, 1996. 100p. Dissertação (Mestrado em Agronomia) - Universidade Estadual Paulista, 1996.

GOMIDE, J.A. Fisiologia do crescimento livre de plantas forrageiras. In: PEIXOTO, A.M.; MOURA, J.C.; FARIA, V.P. (Eds.) Pastagens: Fundamentos da exploração racional. Piracicaba: Fundação de Estudos Agrários Luiz de Queiroz, 2.ed. 1994. p.1-14.

GOMIDE, J.A.; QUEIROZ, D.S. Valor alimentício das Brachiarias. In: SIMPÓSIO SOBRE O MANEJO DA PASTAGEM, 11., 1994, Piracicaba.Anais...Piracicaba: Fundação de Estudos Agrários Luiz de Queiroz, 1994. p.223-248.

GUERRA, A.F.; ANTONINI, J.C. dos A; SILVA, D.B.; RODRIGUES, G.C. Manejo de irrigação e fertilização nitrogenada para a cultura do milho. In: RELATÓRIO TÉCNICO ANUAL DO CENTRO DE PESQUISA AGROPECUÁRIA DOS CERRADOS, 1991 A 1995, p.97-98, 1997.

GUILHERME, L.R.G.; VALE, F.R.; GUEDES, G.A.A. Fertilidade do solo: dinâmica e disponibilidade de nutrientes. Lavras: Escola Superior de Agricultura de Lavras, 1995. $171 \mathrm{p}$.

KRAMER, P. Water relations of plants. New York: Academic Press, 1983. 489p.

LANGER, R.H.M. Tillering in herbage grasses. Herbage Abstrat, v.33, n.3, p.141-148, 1963.

NABINGER, C. Princípios da exploração intensiva de pastagens. In: SIMPÓSIO SOBRE MANEJO DE PASTAGENS, 13., 1997, Piracicaba. Anais... Piracicaba: Fundação de Estudos Agrários Luiz de Queiroz, 1997. p.15-95. 
NORTON, N.W. Differences between species, in forrage quality. In: HACKER, J.B. (Ed.) Commonwealth Agricultural. Bureaux:UK. 1982. p. 89-110.

OLIVEIRA, M.A.; PEREIRA, O.P.; MARTINEZ y HUAMAN, C.A. ET AL. Características morfogênicas e estruturais do capim-bermuda Tifton 85 (Cynodon spp) em diferentes idades de rebrota. Revista Brasileira de Zootecnia, v.29, n.6, p.1939-1948, 2000.

PEDREIRA, C.G.S. Avaliação de novas gramíneas do gênero Cynodon para a pecuária do sudeste dos Estados Unidos. In: WORKSHOP SOBRE O POTENCIAL FORRAGEIRO DO GÊNERO CYNODON, 1996, Juiz de Fora.Anais... Juiz de Fora: EMBRAPA-CNPGL, 1996. p.111-125.

PEDREIRA, C.G.S; NUSSIO, L.G.; SILVA, S.C. Condições edafo-climáticas para produção de Cynodon spp. Manejo de pastagens de Tifton, Coast-cross e estrela. In: SIMPÓSIO SOBRE MANEJO DA PASTAGEM, 15., 1998, Piracicaba. Anais... Piracicaba: Fundação de Estudos Agrários Luiz de Queiroz, 1998. p.23-54.
RIBEIRO, K.G. Rendimento forrageiro e valor nutritivo do capim-Tifton 85, sob diferentes doses de nitrogênio e idades de rebrota, e na forma de feno, com bovinos. Viçosa, MG: Universidade Federal de Viçosa, 2000. 107p. Tese (Doutorado em Zootecnia) - Universidade Federal de Viçosa, 2000.

VILELA, D.; ALVIM, J.M. Manejo de pastagens do gênero Cynodon: introdução, caracterização e evolução do uso no Brasil. In: SIMPÓSIO SOBRE MANEJO DA PASTAGEM, 15., 1998, Piracicaba. Anais... Piracicaba: Fundação de Estudos Agrários Luiz de Queiroz, 1998. p.23-54.

Recebido em: 08/02/02

Aceito em: 08/08/02 